\title{
Modification of Stokes' Law for a Heated Sphere in Liquid Helium II*
}

\author{
B. E. Springett \\ Harrison M. Randall Laboratory of Physics \\ University of Michigan, Ann Arbor, Michigan \\ (Received October 5, 1970; revised January 8, 1971)
}

It is shown that the counterflow which occurs at low heat inputs in liquid helium below the $\lambda$ point modifies the wake behind a steadily moving sphere. The result is valid for a low Reynolds number and limits on the range of the physical variables are given. The outflow of normal fluid results in an increased drag on the sphere. Some discussion is given of the relevance of this result to experiments on small $\mathrm{H}-\mathrm{D}$ particles in liquid helium.

\section{INTRODUCTION}

Our interest in the motion of a heated sphere moving steadily in He II was motivated by a desire to see if a hydrodynamic explanation for the creation of ion-vortex ring complexes ${ }^{1}$ could be formulated. Our interest was further stimulated by the work of Hall, ${ }^{2}$ who measured the reaction pressure in counterflow, and by the heat-torque work of Penney, ${ }^{3}$ Hunt, ${ }^{4}$ and Payne ${ }^{5}$ who demonstrated that super- to normal-fluid conversion can give rise to additional forces on a body immersed in He II.

Basically, the problem is to solve the Navier-Stokes equation for the normal fluid using modified boundary conditions which are brought about by the outward flow of normal fluid from the sphere. In order to take account, at least approximately correctly, of the inertia of the fluid we use the technique of Oseen, ${ }^{6}$ which means we must confine ourselves to a low Reynolds number $R$; that is, $R=2 a U / v_{n} \lesssim 1$, where $a$ is the radius of the sphere, $U$ is the sphere's speed, and $v_{n}$ is the kinematic viscosity of the normal fluid.

Needless to say, the solution of the problem has already been worked out by $\mathrm{Lamb}^{7}$ for the case of regular viscid boundary conditions. The difference between the Oseen and Stokes solutions for the motion of a steadily moving sphere has been made readily apparent by Batchelor. ${ }^{8}$ Briefly, the difference resides in the behavior of the fluid at infinity. In the Stokes solution the flow is

*Work supported in part by the U.S. Atomic Energy Commission. 
symmetric about a plane passing through the center of the sphere and perpendicular to its motion, and is everywhere outward. In the Oseen solution the symmetry no longer exists and at large $r$ the flow is radially outward except for a wake described by a paraboloid of revolution where the flow is inward. Both solutions yield the Stokes formula for the $\operatorname{drag}, 6 \pi a v_{n} \rho_{n} U$, where $\rho_{n}$ is the normalfluid density. This drag is, in fact, equal to $\rho_{n} U$ times the inward volume flux in the wake at large $r$. From this point of view it is easy to see why the drag should be modified if we heat the sphere to produce counterflow.

Lastly, the counterflow also modifies the pressure distribution over the sphere due to the inviscid superfluid.

\section{THE OSEEN SOLUTION}

We start with the Navier-Stokes equation for an incompressible fluid, in which the motion of the sphere is steady in time.

$$
\frac{\partial \mathbf{V}_{n}}{\partial t}+\left(\mathbf{V}_{n} \cdot \nabla\right) \mathbf{V}_{n}=-\left(1 / \rho_{n}\right) \nabla P+v_{n} \nabla^{2} \mathbf{V}_{n}
$$

where $\mathrm{V}_{n}$ is the normal-fluid velocity evaluated in a coordinate system fixed in the fluid, and $P$ is the pressure. We take the sphere to be moving with velocity $-U \mathbf{i}$, where $\mathbf{i}$ is a unit vector in the $x$ direction (which is also the $\theta=0$ direction), and $U$ is the speed of the sphere. For slow, steady motion we may approximate $\partial \mathbf{V}_{n} / \partial t$ by $U \mathbf{i} \cdot \nabla \mathbf{V}_{n}$. Neglecting terms $0\left(V_{n}^{2}\right)$, we obtain

$$
\begin{aligned}
U(\mathbf{i} \cdot \nabla) \mathbf{V}_{n} & =-\left(1 / \rho_{n}\right) \nabla P+v_{n} \nabla^{2} \mathbf{V}_{n} \\
\nabla \cdot \mathbf{V}_{n} & =0
\end{aligned}
$$

Notice that the equation so obtained is equivalent to stopping the sphere and treating the fluid as moving by it, when $\mathbf{V}_{n}$ is a small correction to the fluid velocity at large distances. Equation (2) yields $\nabla^{2} P=0$, so a particular solution is obtained by writing $P=\rho_{n} U \mathbf{i} \cdot \nabla \phi+P_{0}$, where $P_{0}$ is the pressure at infinity. The contribution of this solution to $\mathbf{V}_{n}$ is then $\mathbf{v}=-\nabla \phi$, where $\phi$ satisfies

$$
\nabla^{2} \phi=0
$$

Consequently, since the flow has symmetry about the $x$ axis,

$$
\phi=\sum_{m=\infty}^{\infty}\left(A_{m} r^{m}+B_{m} r^{-m-1}\right) P_{m}(\cos \theta)
$$

If we consider only the boundary condition at infinity where $\mathbf{v}=0$, we see that $A_{0}$ does not enter and $A_{1}=0$.

To complete the solution we write $\mathbf{V}_{n}=\mathbf{v}+\mathbf{u}$ and we find that $\mathbf{u}$ must satisfy

$$
\begin{aligned}
{\left[\nabla^{2}-\left(U / v_{n}\right) \mathbf{i} \cdot \nabla\right] \mathbf{u} } & =0 \\
\nabla \cdot \mathbf{u} & =0
\end{aligned}
$$


If we examine $\nabla_{\Lambda} \mathbf{u}=$ const., we see that this represents circles about the $x$ axis and so we may represent $\nabla_{\Lambda} \mathbf{u}$ as the curl of some vector in the $x$ direction which depends on $(r, \theta)$ only. Let us write

$$
\nabla_{\Lambda} \mathbf{u}=-\nabla_{\Lambda} \chi(r, \theta) \mathbf{i}=\mathbf{i}_{\Lambda} \nabla \chi
$$

Now we take the curl of (5), substitute (6), and arrive at

$$
\left[\nabla^{2}-\left(U / v_{n}\right) \mathbf{i} \cdot \nabla\right] \chi=0
$$

In consequence of the definition of $\mathbf{u}, \chi$ must vanish at infinity, which gives the allowed solutions of (7). Equation (7) may be rewritten in the form

$$
\left(\nabla^{2}-\kappa^{2}\right) e^{-\kappa \mathbf{i} \cdot \mathbf{r}} \chi=0
$$

where $\kappa=U / 2 v_{n}$. The solutions of $(8)$ which vanish at infinity are

$$
\chi=e^{\kappa r \cos \theta} \sum_{l=0}^{\infty} E_{l} k_{l}(\kappa r) P_{l}(\mu)
$$

where the $k_{l}$ are modified spherical Bessel functions of the third kind, which go to zero as $r \rightarrow \infty$.

Lastly we need to express $\mathbf{u}$ in terms of $\chi$. From (5) and (6) we have

$$
\begin{aligned}
2 \kappa \mathbf{i} \cdot \nabla \mathbf{u} & =\nabla^{2} \mathbf{u}=-\nabla_{\Lambda}\left(\nabla_{\Lambda} \mathbf{u}\right) \\
& =-\mathbf{i}\left(\nabla^{2} \chi\right)+(\mathbf{i} \cdot \nabla) \nabla \chi \\
& =-2 \kappa \mathbf{i}(\mathbf{i} \cdot \nabla \chi)+(\mathbf{i} \cdot \nabla) \nabla \chi
\end{aligned}
$$

So, finally,

$$
\mathbf{u}=-\chi \mathbf{i}+\left(\frac{1}{2} \kappa\right) \nabla \chi
$$

We see then that the full solution is very complicated and in the next section where we discuss the boundary conditions on the sphere we shall also adopt the simplest form of (4) and (9), which is what limits the present solution to a low Reynolds number.

\section{BOUNDARY CONDITIONS}

In the two-fluid model for He II entropy is associated only with normal fluid. Hence if the sphere gives off heat at the rate of $4 \pi a^{2} \dot{Q}$, we have

$$
\dot{Q}=\rho S T v_{n}
$$

where $\rho$ is the total fluid density, $S$ is the entropy per $g, T$ is the absolute temperature, and $v_{n}$ is the velocity of the normal fluid away from the sphere evaluated at $r=a$. In the absence of interaction between the two fluids we must also have $\rho_{n} v_{n}+\rho_{s} v_{s}=0$, so that

$$
v_{s}=\left(\rho_{n} / \rho_{s}\right) \dot{Q}(\rho S T)^{-1}
$$

at $r=a$, and is radially inward. The boundary condition on the surface of the 
sphere then becomes

$$
\mathbf{V}_{n}=-U \mathbf{i}+\mathbf{v}_{n}
$$

which reduces to the usual boundary condition if $\dot{Q}=0$.

Since (13) involves only $\sin \theta$ and $\cos \theta$ we shall keep only those terms in (4) and (9) which yield such terms. In addition, we shall now explicitly assume a low Reynolds number so that we may take $\kappa a$ small and expand the exponential in (9). Also, $\dot{Q}$ must be such that there is no turbulent counterflow.

Applying the boundary conditions we find

$$
\begin{aligned}
-U \cos \theta+v_{n}= & B_{0} a^{-2}+2 B_{1} a^{-3} \cos \theta+\frac{1}{2} E_{0} k_{0}^{\prime}(\kappa a) \\
& -\frac{1}{2} E_{0}\left[k_{0}(\kappa a)-(\kappa a) k_{0}^{\prime}(\kappa a)\right] \cos \theta
\end{aligned}
$$

and

$$
U \sin \theta=B_{1} a^{-3} \sin \theta+\frac{1}{2} E_{0} k_{0}(\kappa a) \sin \theta
$$

where $k_{0}^{\prime}$ denotes $\partial k_{0} / \partial(\kappa r)$.

Upon equating the coefficients of the angular terms in (14) and (15) the following values for the constants result :

$$
\begin{aligned}
& B_{0}=v_{n} a^{2}-\left[U a^{2} k_{0}^{\prime}(\kappa a) / k_{0}(\kappa a)\right]\left[1-(\kappa a) k_{0}^{\prime}(\kappa a) / 3 k_{0}(\kappa a)\right]^{-1} \\
& B_{1}=U a^{3}-U a^{3}\left[1-(\kappa a) k_{0}^{\prime}(\kappa a) / 3 k_{0}(\kappa a)\right]^{-1} \\
& E_{0}=\left(2 U / k_{0}(\kappa a)\right)\left[1-(\kappa a) k_{0}^{\prime}(\kappa a) / 3 k_{0}(\kappa a)\right]^{-1}
\end{aligned}
$$

These expressions are considerably simplified if we take the limit $\kappa a \rightarrow 0$. We get

$$
\begin{aligned}
& B_{0}=v_{n} a^{2}+3 U a / 4 \kappa \\
& B_{1}=U a^{3} / 4 \\
& E_{0}=(3 U \kappa a / \pi)\left(1-\frac{\kappa a}{4}\right) e^{\kappa a}
\end{aligned}
$$

The full expression for the radial and tangential components of the velocity field in terms of the coefficients are as follows:

$$
\begin{aligned}
& V_{n r}=B_{0} r^{-2}+2 B_{1} r^{-3} \cos \theta+\frac{1}{2} E_{0}\left[k_{0}^{\prime}(\kappa r)-k_{0}(\kappa r) \cos \theta\right] e^{\kappa r \cos \theta} \\
& V_{n \theta}=B_{1} r^{-3} \sin \theta+\frac{1}{2} E_{0} k_{0}(\kappa r) \sin \theta e^{\kappa r \cos \theta}
\end{aligned}
$$

We shall not go into great detail at this point but simply note that for $r \sim a$ we recover the Stokes solution for the case $\dot{Q}=0$, and that, again for $\dot{Q}=0$, the behavior of $V_{n r}$ and $V_{n \theta}$ at large $r$ can be divided in to two cases.

Case 1

$$
\begin{aligned}
\theta & \gtrsim \pi / 2 \quad r \gg 4 a / R \\
V_{n r} & \approx B_{0} r^{-2} \approx 3 U a^{2} / R r^{2} \\
V_{n \theta} & \approx B_{1} r^{-3} \sin \theta \approx \frac{U}{4}\left(\frac{a}{r}\right)^{3} \sin \theta
\end{aligned}
$$


i.e., the flow is almost purely radial and outward from the sphere. These relations become more exact as $\theta \rightarrow \pi$.

Case 2

$$
\begin{aligned}
\theta & \sim 0 \quad r \gg 4 a / R \\
V_{n r} & \approx 6\left(U a^{2} / R r^{2}\right)-\frac{3}{2}(U a / r) \\
V_{n \theta} & \approx \frac{3}{4}(U a / r) \sin \theta
\end{aligned}
$$

i.e., the flow remains almost radial but is now in toward the sphere. It is this flow of fluid which constitutes the wake. The dividing line between the two regions is given by, for $r$ large,

$$
\kappa r(\cos \theta+1) \exp [\kappa r(\cos \theta-1)]=1
$$

This equation has the property that as $r \rightarrow \infty, \theta \rightarrow 0$. From the boundary condition (14) we see in the absence of $v_{n}$ that flow is toward the sphere for $\theta<\pi / 2$. An approximate solution to $(21)$ is $\theta \sim(2 / \kappa r)^{1 / 2}$. If we now relax the condition $\dot{Q}=0$, (21) becomes modified by the addition of a term $2 a v_{n} / 3 v_{n}$ to the right-hand side. A term such as this can easily be quite large in helium and increasing the righthand side of (21) has the effect of decreasing $\theta$ for a given $r$. Furthermore, for $r \sim a$, the presence of $v_{n}$ decreases the area of the sphere over which fluid is flowing inward. As $v_{n}$ is increased we reach a point where no fluid flows inward for $r \sim a$, but still flows inward for large $r$. This point is reached when $v_{n}=U$. If we consider the $\theta=0$ axis where $V_{n \theta}=0$, we see that when $v_{n}=U$ a stagnation point develops away from the boundary of the sphere, which moves along with the sphere.

\section{VORTICITY GENERATION}

If we examine the vorticity, $\omega=\nabla_{\Lambda} \mathbf{V}_{n}$, we find that at large distances it is confined to the wake. The moving sphere creates vorticity at its surface which is left behind as the sphere moves on diffusing outward into the wake region. ${ }^{9}$ It should be noted that because of the definition of $\omega, v_{n}$ contributes nothing to the vorticity. Vorticity may also be convected by the fluid and it is the balance between diffusion and convection which determines the shape of the wake. This is easily seen from our approximate solution to $(21), \theta \sim(2 / \kappa r)^{1 / 2}=\left(4 v_{n} / 2 U r\right)^{1 / 2}$, which indicates that the wake becomes narrower the larger $U$. Consequently, we argue that when $v_{n}$ is such that a stagnation point separates from the sphere, convection causes a build up of vorticity in the neighborhood of the stagnation point.

So far we have allowed no interaction between normal and superfluid. Presumably, in order to create vorticity in the superfluid it must interact with the normal fluid. Similar considerations apply to creation of vortex lines in a rotating bucket. If such a bucket is set into rotation below the $\lambda$ point vorticity is created at the wall in the normal fluid, which spreads in time throughout the bucket prior to the normal fluid coming into solid-body rotation. If the two-fluid equations are coupled by a Gorter-Mellink ${ }^{10}$ mutual friction term, vorticity can be induced in the superfluid. We postulate a similar induction of vorticity in this case. 


\section{DRAG FORCE ON THE SPHERE}

The equations we have developed for the radial and tangential components of the induced fluid velocity reduce to those of the regular Stokes solution near the sphere. To calculate the drag on the sphere we use these velocity components to evaluate the stress tensor over the surface of the sphere. Thus, the force per unit area on the sphere is ${ }^{11}$

$$
F_{i}=-\sigma_{i k} n_{k}=P n_{i}-\sigma_{i k}^{\prime} n_{k}
$$

where $\mathbf{n}$ is a unit vector outward from the fluid, normal to the surface of the sphere. Since we are dealing with a sphere this reduces to

$$
\left.\begin{array}{l}
F_{r}=P-\sigma_{r r}^{\prime} \\
F_{\theta}=-\sigma_{r \theta}^{\prime}
\end{array}\right\} \text { at } r=a
$$

To calculate the drag force $\mathbf{F}_{D}$ we need to project these components onto the direction of motion of the sphere and integrate over the surface of the sphere, i.e.,

$$
\begin{aligned}
\mathbf{F}_{D} & =\mathbf{i} \int_{s} d S\left(-P \cos \theta+\sigma_{r r}^{\prime} \cos \theta-\sigma_{r \theta}^{\prime} \sin \theta\right)_{r=a} \\
& =2 \pi a^{2} \mathbf{i} \int_{1}^{-1}\left(P \cos \theta-\sigma_{r r}^{\prime} \cos \theta+\sigma_{r \theta}^{\prime} \sin \theta\right)_{r=a} d(\cos \theta)
\end{aligned}
$$

Evaluating $\sigma_{r r}^{\prime}$ and $\sigma_{r \theta}^{\prime}$ we find, in terms of the coefficients (16), that ${ }^{12}$

$$
\mathbf{F}_{D}=\frac{4 \pi}{3} \rho_{n} U\left[B_{0}-3 a^{2} E_{0} k_{0}^{\prime}(\kappa a) i_{1}(\kappa a) /(\kappa a)\right] \mathbf{i}
$$

where $i_{1}$ is a modified spherical Bessel function of the first kind. At this point we take the value of (25) in the limit $\kappa a \rightarrow 0$ and $\dot{Q} \neq 0$, which after some algebra we find to be

$$
\mathbf{F}_{D}=\left\{\frac{4 \pi}{3} a^{2} \rho_{n} v_{n} U+6 \pi a \eta U\left(1+\frac{3 a U}{8 v_{n}}\right)\right\} \mathbf{i}
$$

That is, we recover the drag force correct up to a term $O\left(a^{2}\right)$ for $\dot{Q}=0$, and we see that an extra term appears when $\dot{Q} \neq 0$. This extra term comes from the coefficient $B_{0}$, which in turn comes solely from the pressure term in (24). [It integrates to zero for the other two terms in (24).] So the physical origin of the extra drag term resides in the fact that the outflow of normal fluid from the sphere modifies the pressure distribution over the sphere.

We have, however, omitted to take account of the superfluid component. To do this we recognize that we are working in the limit $\rho_{s} v_{s}+\rho_{n} v_{n}=0$, i.e., no mass transfer is occurring. It is quite trivial to evaluate $\mathbf{v}_{s}$, as we can treat the superfluid as an ideal fluid and find the inward radial flow due to the heat source from the zero-mass-flow condition, with the sphere as the coordinate origin. 
An extra drag on the sphere will result because of superfluid-to-normal-fluid conversion at the sphere's surface. Let us also assume, since it does not complicate the problem very much, that the superfluid is also flowing with pure potential flow at some arbitrary direction with respect to the velocity of the sphere. Let this superfluid velocity be $\mathbf{V}_{s}$ and

$$
-U \mathbf{i} \cdot \mathbf{V}_{s}=U V_{s} \cos \phi
$$

The total superfluid velocity in the vicinity of the sphere will then be composed of $\mathbf{v}_{s}$ due to the heat source, $\mathbf{V}_{s}$ due to the potential flow, plus a part induced by the motion of the sphere. To work out the superfluid velocity components we transform to a frame where the sphere is moving through stationary superfluid at a velocity $\mathbf{U}_{1}=-U \mathbf{i}-\mathbf{V}_{s}$. This means that the sphere is moving at an angle, $\beta$ with respect to $-\mathbf{i}$ defined by

$$
\sin \beta=\left(V_{s} / U_{1}\right) \sin \phi
$$

The evaluation of the superfluid flow in terms of this new direction of motion is then trivial. ${ }^{13}$ We then evaluate the momentum flux tensor for each fluid component, noting that we have already taken account of the normal-fluid pressure in (22). This then allows us to calculate the normal and tangential components of force on the sphere due to the momentum flux into the surface. Integrating over the surface of the sphere then gives the net drag on the sphere. As we would expect, this procedure gives zero drag due to the normal fluid and the answer for the superfluid is

$$
\mathbf{F}_{s}=4 \pi \rho_{s} a^{2} v_{s}\left(U \mathbf{i}+\mathbf{V}_{s}\right)
$$

i.e., if $U=0$, the superfluid drags the sphere along. In fact, in this case our result coincides with that of Penney and Hunt. ${ }^{3}$ If $V_{s}=0$, the superfluid gives rise to an extra term in the Stokes drag. So our result for the drag force on a heated sphere in helium II is

$$
\begin{aligned}
\mathbf{F}_{D} & =\left\{4 \pi a^{2} U\left(\frac{1}{3} \rho_{n} v_{n}+\rho_{s} v_{s}\right)+6 \pi a \eta U\left(1+3 a U / 8 v_{n}\right)\right\} \mathbf{i}+4 \pi a^{2} \rho_{s} v_{s} \mathbf{V}_{s} \\
& =6 \pi a \eta U\left(1+3 a U / 8 v_{n}+8 a \dot{Q} / 9 \rho S T v_{n}\right) \mathbf{i}+4 \pi a^{2} \rho_{s} v_{s} \mathbf{V}_{s}
\end{aligned}
$$

In (29), the first correction in the bracket is just $\frac{3}{16} R$ and the second correction in the bracket is an effective Prandtl number $P^{*}$ for liquid helium below the $\lambda$ point, since $a \dot{Q} / T$ has the dimensions of thermal conductivity and $S$ has the same dimensions as specific heat. Thus, we may write $\chi^{*}=a \dot{Q} / \rho S T$ for the thermometric conductivity; and then $P^{*}=v_{n} / \chi^{*}$, so that the correction to the Stokes' drag in the absence of superfluid flow is $\left(1+\frac{3}{16} R+\frac{8}{9} P^{*-1}\right)$.

\section{DISCUSSION}

There are several situations which are of possible experimental interest. We shall only consider two such situations in order to see what (29) means in terms of the motion of a microsphere. We take the following situation which corresponds 
roughly to the experiments performed by Chung and Critchlow ${ }^{14}$ and is related to those done by Steyert et al. ${ }^{15}$ The superfluid is made to move with velocity $V_{s}$ in plane-parallel flow with the normal fluid stationary. Small spheres $\left(a \sim 10^{-2}\right.$ $\mathrm{cm}$ ) of neutral buoyancy are suspended in the fluid. The spheres emit heat at a rate of $1 \mathrm{~mW} \mathrm{~cm}{ }^{-2} \mathrm{sec}^{-1}$. (This is not unreasonable if the source of heat is absorbed room-temperature radiation and the spheres are a hydrogen-deuterium mixture). Initially, we will ignore the fact that the velocity necessary to achieve a Reynolds number of $\sim 1$, which is the limit of validity of the Oseen solution, is less than $5 \times 10^{-2} \mathrm{~cm} \mathrm{sec}^{-1}$ above $1.5 \mathrm{~K}$, and assume we can ignore the Oseen correction to the normal-fluid drag. We then find that the particle velocity is given by

$$
U=V_{s}\left(\frac{3}{2} P^{*}+\frac{4}{3}\right)^{-1}
$$

Thus when $P^{*} \sim 0, U \sim V_{s}$ and in the same direction, and for $P^{*} \gg 1, U \rightarrow 0$. A plot of the quantity in brackets vs. temperature is shown in Fig. 1 for the above conditions. The data for the figure are taken from Wilks. ${ }^{16}$ Over a considerable

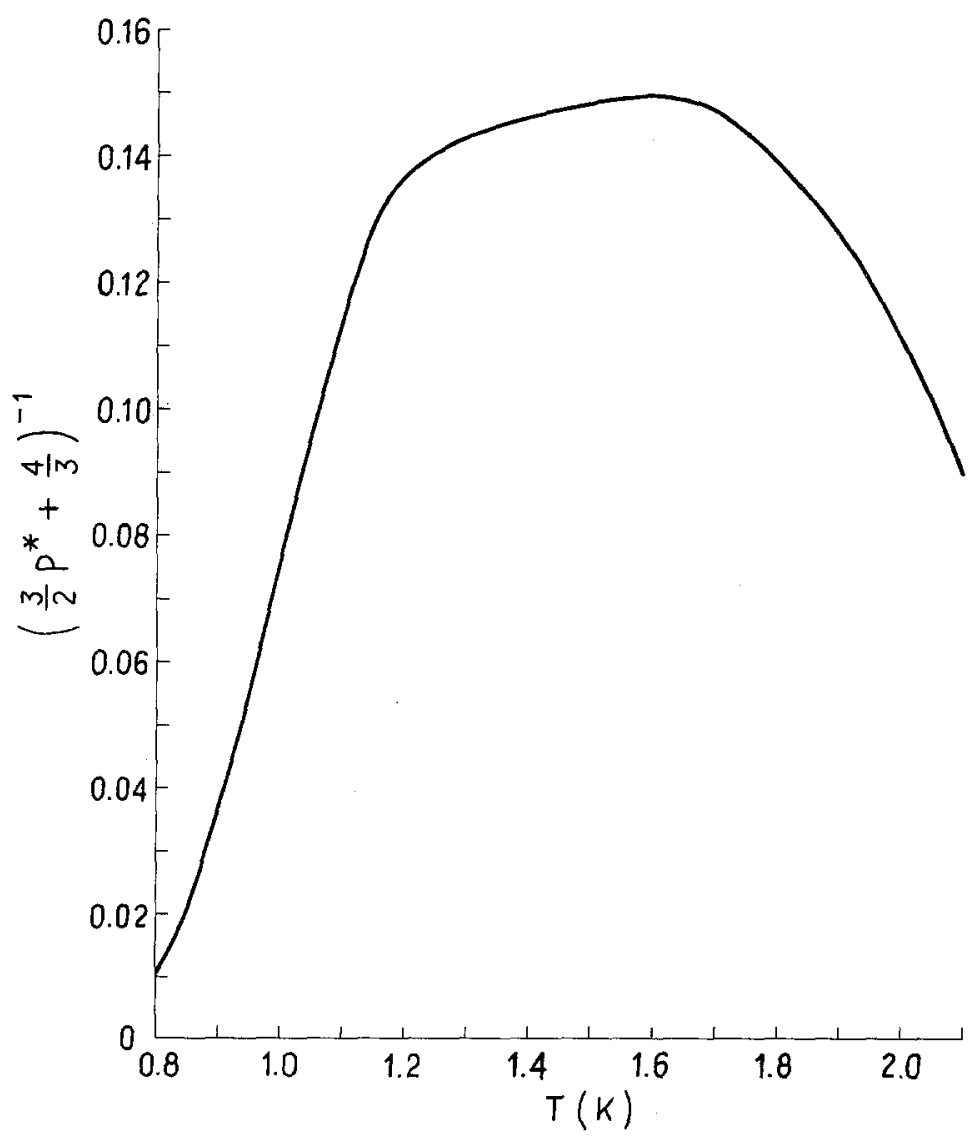

Fig. 1. A plot of the quantity $\left(\frac{3}{2} p^{*}+\frac{4}{3}\right)^{-1}$ appearing in Eq. (30) vs. temperature for the conditions given in the text. 
range of temperature $P^{*} \sim 4$, and we would expect $U \sim V_{s} / 7$. If $\dot{Q}$ were a factor of 10 bigger we would expect $U \sim V_{s} / 2$. Similarly, $U$ is increased if $a$ is made large. This corresponds reasonably well with what was observed by Chung and Critchlow. Above $V_{s} \sim 0.1 \mathrm{~cm} \mathrm{sec}{ }^{-1}$ they found $U \geq V_{s}$. Our criterion for significant convection of vorticity by normal-fluid motion into the sphere is $v_{n} \approx U$. At this point we expect the normal-fluid flow about the sphere to become quite complicated and its effect upon the motion is uncertain, though it is likely that the normal-fluid drag will be reduced due to dragging of the normal fluid by the superfluid. The conditions we have used give $U \sim 5 \times 10^{-2} \mathrm{~cm} \mathrm{sec}^{-1}$ for this effect to set in which by virtue of (30) implies $V_{s} \sim 0.35 \mathrm{~cm} \mathrm{sec}^{-1}$. Chung and Critchlow saw indications of turbulent flow with $U \sim V_{s}$ for $0.1<V_{s}<0.3 \mathrm{~cm} \mathrm{sec}^{-1}$.

The second situation we wish to examine is that of a sphere being moved by an external force through stationary liquid helium. Again assuming $R$ such that we can ignore corrections to the drag force due to the normal fluid, the terminal velocity $U_{s}$ of the sphere will be given by the usual Stokes Law if the sphere is unheated. If the sphere is heated the terminal velocity $U_{H}$ is given by

$$
U_{H}=U_{s} P^{*}\left(P^{*}+\frac{8}{9}\right)^{-1}
$$

If $P^{*}$ is large no effect will be seen. If $P^{*}$ is made small $U_{H}$ becomes considerably reduced. A small sphere may be heated by embedding in it a radioactive material. For example, if $1 \mu \mathrm{Ci}$ of an $\alpha$ emitter is embedded in a $10-\mu$ sphere and all the energy liberated per disintegration is emitted as heat from the sphere's surface this amounts to $\sim 1 \mathrm{~mW} \mathrm{~cm}{ }^{-2} \mathrm{sec}^{-1}$ and $P^{*}$ is large. However, if we embed $100 \mu \mathrm{Ci}, P^{*}$ is about 0.5 and $U_{H} \sim U_{s} / 3$. Under these conditions, then, treated and untreated spheres should show a considerable sedimentation effect.

\section{CONCLUSION}

We have developed an expression for the drag on a heated sphere which is a good approximation up to a Reynolds number of about unity. In terms of the parameters of a given situation this means the sphere's velocity $U$ must be less than $v_{n} / 2 a$. This in turn means that the effective Prandtl number $P^{*}$ must be greater than about two. We have discussed two experimental situations and shown that the two-fluid counterflow generated at the sphere can lead to unwanted complications in an experimental situation. We have shown that the normalfluid motion as well as the superfluid motion must be taken into account in determining the additional drag forces on a moving, heated object in helium II.

\section{REFERENCES}

1. S. Cunsolo and B. Maraviglia, Phys. Rev. 187, 292 (1969), and references therein.

2. H. E. Hall, Proc. Phys. Soc. (London) A67, 485 (1954).

3. R. Penney, Phys. Fluids 10, 2147 (1967); R. Penney and A. W. Overhauser, Phys. Rev. 164, 268 (1967); R. Penney and T. K. Hunt, Phys. Rev. 169, 228 (1968).

4. T. K. Hunt, Phys. Rev. 170, 245 (1968).

5. R. S. Payne, Phys. Rev. Letters 24, 710 (1970). 
6. C. W. Oseen, Arkiv Mat. Astron. Fysik 6, No. 29 (1910).

7. H. Lamb, Phil. Mag. 21, No. 6, 112 (1911).

8. G. K. Batchelor, An Introduction to Fluid Mechanics (Cambridge University Press, Cambridge, England, 1967), pp. 231, 242.

9. Ibid., p. 243.

10. C. J. Gorter and J. H. Mellink, Physica 15, 285 (1949).

11. L. D. Landau and E. M. Lifshitz, Fluid Mechanics (Addison-Wesley, Reading, Mass., 1959), p. 50.

12. Ibid., p. 52.

13. Ibid., p. 25.

14. D. Y. Chung and P. R. Critchlow, Phys. Rev. Letters 14, 892 (1965); D. Y. Chung, in Proceedings of the Eleventh International Conference on Low Temperature Physics, J. F. Allen, D. M. Finlayson, and D. M. McCall, eds. (University of St. Andrews Printing Department, St. Andrews, Scotland, 1969).

15. W. A. Steyert, R. D. Taylor, and T. A. Kitchens, Phys. Rev. Letters 15, 546(1965).

16. D. Wilks, The Properties of Liquid and Solid Helium (Clarendon Press, Oxford, England, 1967), Table A1 and A5. 